\title{
A Novel Implementation of Chiral Structure in Computer Honeycomb Diamond Networks Topology
}

\author{
Mr. Susheel George Joseph M.C.A, M.Tech, M.Phil (Cs) \\ (Associate Professor, Department of M.C.A, Kristu Jyoti College of Management and Technology, \\ Changanassery, Kerala)
}

\begin{abstract}
This project deals with the efficiency of the Chiral structure in Honeycomb Diamond network. Honeycomb and diamond networks have been proposed as alternatives to mesh and torus architectures for parallel processing. When wraparound links are included in honeycomb and diamond networks, the resulting structures can be viewed as having been derived via a systematic pruning scheme. Honeycomb networks are more efficient than mesh connected systems in terms of degree, diameter, and cost and bisection bandwidth. By embedding Chiral structure in Honeycomb Diamond network we are enhancing the efficiency of Network and compare this efficiency with other networks. Comparing the resultant structure's efficiency in high traffic situations and to find best suitable area where this can be useful.
\end{abstract}

Keyword: Honeycomb, Chiral, Pruning, Diamond Networks

\section{Introduction And Scope}

Presently we have many types of networks with different structures. Still we have some drawbacks in these networks like handling heavy traffic situations, efficiency lack, efficient usage of bandwidth etc. Recently they use the concepts of honeycomb networks in the networking. But still they are facing some problems. So now we are enhancing the efficiency of new structure in the Diamond network to overcome some of the above problems.

Presently we are embedding a new structure named Chiral structure into the Honeycomb Diamond networks. We are enhancing the efficiency of Network and compare this efficiency in high traffic situations and to find the best suitable area for this network. The comparison should be done on basis of cost, degree, and diameter. Honeycomb and diamond networks have been proposed as alternatives to mesh and torus architectures for parallel processing.

Problem:

\section{Formulation Of The Problem And Analysis}

At present, we are having different networks which face some problems in high traffic situations. Now we are embedding a new structure into networks and compare the efficiency of the resultant network with other networks available. We analyze the efficiency of resultant structure in terms of degree, diameter, and cost and bisection bandwidth.

\section{Solution:}

We are embedding Chiral structure into Diamond networks to check the handling capacity of resultant network in high traffic situations. We should analyze the best area which suits the resultant network. The following is the chiral structure which we are going to embed in Diamond network.

The efficiency and structure properties are analyzing and learning the Network simulation

Network simulation is a technique where a program models the behavior of a network either by calculating the interaction between the different network entities (hosts/packets, etc.) using mathematical formulas, or actually capturing and playing back observations from a production network. The behavior of the network and the various applications and services it supports can then be observed in a test lab; various attributes of the environment can also be modified in a controlled manner to assess how the network would behave under different conditions.

A network emulator allows users to introduce real devices and applications into a test network (simulated) that alters packet flow in such a way as to mimic the behavior of a live network. Live traffic can pass through the simulator and be affected by objects within the simulation.

The typical methodology is that real packets from a live application reach the emulation server (where the virtual network is simulated. The real packet gets modulated into a simulation packet. The Simulation packet gets demodulated into real packet after experiencing effects of loss, errors, delay, jitter etc., thereby transferring these network effects into the real packet. Thus it is as-if the real packet flowed through the real networks but in reality it flowed through the simulated network. 
Emulation is widely used in the design stage for validating communication networks prior to deployment.

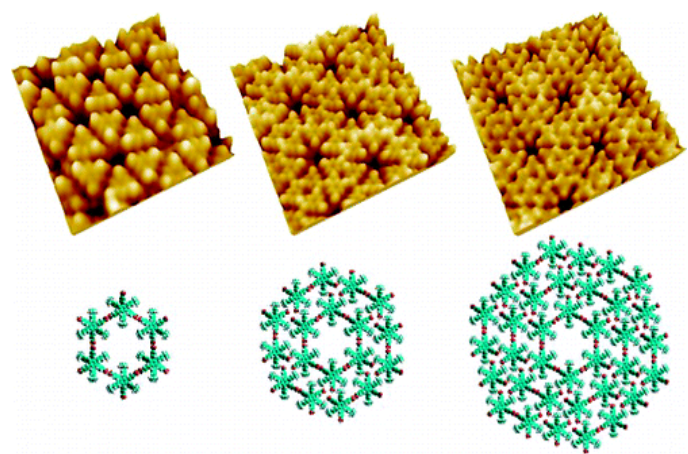

Figure 1 Chiral structures of various dimensions

\section{Modules}

Developing Honeycomb with Diamond network

Here we are forming Honeycomb Diamond network by clustering different Diamond networks. The following figure shows the Honeycomb diamond network.

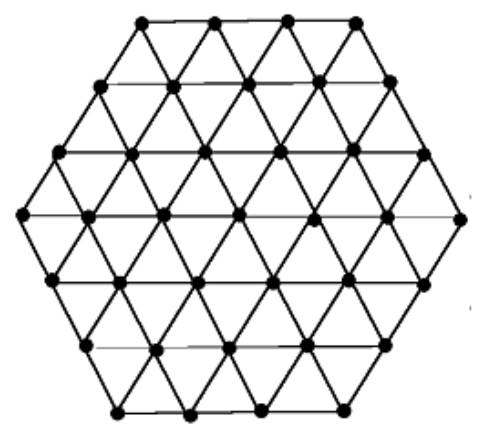

Figure 2 Honeycomb Diamond Network

Honeycomb and diamond networks have been proposed as alternatives to mesh and torus architectures for parallel processing. When wraparound links are included in honeycomb and diamond networks, the resulting structures can be viewed as having been derived via a systematic pruning scheme applied to the links of 2D and $3 \mathrm{D}$ tori, respectively. The removal of links, which is performed along a diagonal pruning direction, preserves the network's node-symmetry and diameter, while reducing its implementation complexity and VLSI layout area. Because pruning reduces the node degree without increasing the network diameter, the pruned networks have an advantage when the degree-diameter product is used as a figure of merit. Additionally, if the reduced node degree is used as an opportunity to increase the link bandwidths to equalize the costs of pruned and unpruned networks, a gain in communication performance may result.

\section{Implementing Chiral Structure into Diamond network}

In this module we are embedding Chiral structure into Diamond networks. The Chiral structures are connected in the form of Diamond. The importance of Chiral structure is, the no of free nodes is more. The following figure shows the Chiral structure.

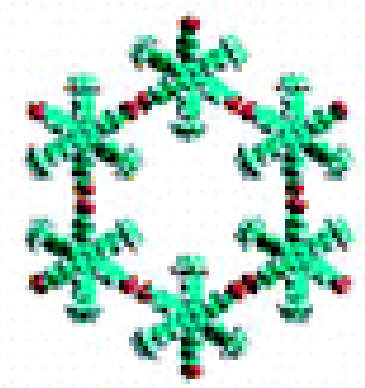

Figure3. Chiral structure implemnted 


\section{Embedding both Modules}

In this module we embed the both Chiral Diamond structure and form Honeycomb Diamond network with that. The resulted network is tested in case of high traffic situations in terms of efficiency and cost.

\section{Embed the Chiral structure into Diamond network}

We should embed Chiral structure into Diamond networks. The Chiral structures are connected in the form of Diamond.

\section{Checking efficiency of resultant in different areas}

We enhance the efficiency in high traffic situations and to find the best suitable area for this network.

\section{Compare with other networks.}

We enhance the efficiency of Network and compare this efficiency with other networks.

\section{Striped networks and other hierarchical structures in Am BmCn (2m + n)-miktoarm star terpolymer melts Chiralization of Diamond Nets: Stretchable Helices and Chiral and Achiral Nets with Nearly Identical Unit Cells}

Recently the formation of a "striped" diamond or zincblende structure has been reported or predicted in various different but similar self-assembled soft matter systems. The first report was an experimental identification in a blend of $\mathrm{ABC}$ three-miktoarm star terpolymers and linearC homopolymers . Later two simulation studies predicted the same structure in other related systems, one from self-assembled star polyphiles with unequal arm volumes and one in a polymertethered nanoparticle system. Among other things, this particular structure is interesting due to its potential as a self-organized photonic crystal where diamond-type structures are especially well suited because of their superior photonic band-gap properties. In general, the selfassembly of new unusual morphologies is a growing field in contemporary soft matter research driven by the ability to synthesize increasingly complex molecular architectures. Examples of recent complex ordered structures based on star-shaped molecules include (apart from the above mentioned) multicolored perforated lamellae, hierarchical lamellae, and novel network structures, all in various miktoarm star systems as well as a range of complicated cylindrical patterns in polyphilic bolaamphiphiles. In this article, a conceptually simple road to obtain striped bicontinuous analog morphologies and other complex hierarchical structures is shown using computer simulations, and the importance of the molecular topology is emphasized as a way to control the interfacial curvature in liquid-crystalline soft matter assemblies. Ultimately we conclude that the molecular architecture should be considered as important a structural variable as composition and interaction parameters which in simpler linear copolymer systems are the overwhelmingly dominant characteristics.

Stretchable diamond nets: Homochiral and achiral diamond nets with nearly identical unit cells have been found in a family of 3D framework materials containing flexible helical substructures (see picture; $\mathrm{Cd}$ green, $\mathrm{O}$ red, C gray). Such structures raise intriguing prospects for developing general strategies for the direct conversion of achiral four-connected networks into homochiral networks through ligand substitution.

\section{References}

[1]. Behrooz Parhami, Fellow, IEEE, And Ding-Ming Kwai, “'A Unified Formulation Of Honeycomb And Diamond Networks”, IEEE Trans. Parallel And Distributed Systems, 12(1) Jan 2001

[2]. Yutaka Yamada, Hideharu Amano, Michihiro Koibuchi, Akiya Jouraku, Kenichiro Anjo, And Katsunobu Nishimura, “Folded Fat H-Tree: An Interconnection Topology For Dynamically Reconfigurable Processor Array', Embedded And Ubiquitous Computing, International Conference EUC 2004, Aizu-Wakamatsu City, Japan, August 25-27, 2004

[3]. André Dehon, “Compact, Multilayer Layout For Butterfly Fat-Tree', Twelfth Annual ACM Symposium On Parallel Algorithms And Architectures (SPAA 2000, July 9-12, 2000), P. 206--215.

[4]. B. Parhami, D.-M. Kwai., “Comparing Four Classes Of Torus-Based Parallel Architectures: Network Parameters And Communication Performance', Mathematical And Computer Modeling, Vol. 40, Nos. 7-8, Pp. 701-720, October 2004.

[5]. Enrique Vallejo, Ramón Beivide, Carmen Martínez, "Practicable Layouts For Optimal Circulant Graphs", 13th Euromicro Conference On Parallel, Distributed And Network-Based Processing (PDP'05), February 09 - 12, 2005, Lugano, Switzerland.

[6]. Wai Hong Ho, Timothy Mark Pinkston, University Of Southern California, “'A Methodology For Designing Efficient On-Chip Interconnects On Well-Behaved Communication Patterns", Ninth International Symposium On High-Performance Computer Architecture (HPCA'03), 0208 - 02, 2003, Anaheim, California.

[7]. Terry Tao Ye, Giovanni De Micheli, “Physical Planning For On-Chip Multiprocessor Networks And Switch Fabrics”, IEEE International Conference On Application-Specific Systems, Architectures, And Processors (ASAP'03), 06 24 - 06, 2003, The Hague, The Netherlands.

[8]. Dégila, J., Sansò, B., “A Survey Of Topologies And Performance Measures For Large Scale Networks”, IEEE Communications Surveys And Tutorials Magazine, Fourth Quarter, 6 (3) Pp 2-15, 2004

[9]. Stojmenovic, ${ }^{a}$ honeycomb Networks: Topological Properties And Communication Algorithms ${ }^{\mathbf{o}}$ IEEE Trans. Parallel And Distributed Systems, Vol. 8 Pp. 1836-1842, Oct. 1997.

[10]. Efficient Area Planning For Personal Communication Systems, Yigal Bejerano, Mark .Smith , Joseph Noar, And Nicole Immorlica 